\title{
Influence of curing agents on gelation and exotherm behaviour of an unsaturated polyester resin
}

\author{
RAGHU RAJA PANDIYAN KUPPUSAMY ${ }^{\dagger}$ and SWATI NEOGI* \\ Department of Chemical Engineering, Indian Institute of Technology, Kharagpur 721 302, India \\ ${ }^{\dagger}$ Birla Institute of Technology, Mesra, Ranchi 835 215, India
}

MS received 19 April 2012; revised 24 February 2013

\begin{abstract}
A judicious choice of curing agents such as initiator and promoter and their ratio to the resin can avoid reduced gel-time and shortened exothermic reactions in applications such as liquid composite moulding processes. In this study, effects of different ratio of initiator and promoter to the unsaturated polyester resin on curing of the resin were investigated by measuring gel-time and peak exotherm using ASTM D2471 standards. Methyl ethyl ketone peroxide (MEKP) was used as an initiator and a cobalt salt was employed as an accelerator for the free radical polymerization of curing resin at ambient temperatures. It was observed that the resin gelation starts closely with the initial rise in exotherm temperature and time of gelation decreases with the increase in initiator or accelerator volume proportions. It was also found that the exotherm-peak and rate of temperature rise indicating that the curing rate increases with the initiator or accelerator proportions also increased. A nonlinear regression analysis of all geltime and cure data were performed to quantify the dependence of curing parameters on the volume proportions of accelerator and initiator. Thus, for this polymerization initiation system, the gel-time and cure parameters can be predicted for any initiator and cobalt levels within the ranges studied.
\end{abstract}

Keywords. Unsaturated polyester resin; resin curing; gel time; exotherm behaviour; peroxide initiator.

\section{Introduction}

Resin transfer moulding involves different phenomena such as resin flow, heat transfer and polymerization reactions simultaneously. The resin polymerization reaction leads to phase transformation from viscous liquid to rigid solid with an exothermal effect. The gel time which marks the onset of gelation or curing reaction associated with a sharp increase in viscosity of the resin system constitutes a crucial parameter for the mould fill time. The required component geometry needs to be filled well before the resin gels prior to the initiation of cure cycles. Hence, the prediction of resin gel times and exothermal behaviour enhance complete RTM process cycle to manufacture a composite part. The magnitude of gel times and the exotherm behaviour for a particular resin system can be altered with the ratio of initiators and promoters to the resin and the elevation of applied process temperatures. Unsaturated polyester (UP) resin is one of the most popular thermoset matrices used in advanced polymeric composite structures because of its optimized properties and cost parameters, good processing characteristics, specific physical properties, moderate price and its ability to cure under normal temperatures and pressures during thermal and compression cycles makes it an attractive resin candidate. The curing of UP resin is a free radical polymerization, i.e. highly exothermic in nature in which the resin is

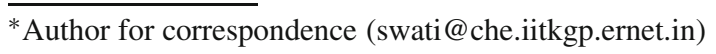

transferred from liquid state into a rigid cross-linked molecular structure that becomes insoluble and infusible.

Physically, curing of resin is the overall transformation from liquid to gel, then from gel to rigid solid as a result of cross linking with a measurable exothermic effect. Gel time marks the formation of an infinite molecular weight $3 \mathrm{D}$ network, which makes sudden irreversible transformation from a liquid resin to a non-flowing gel. Therefore, all moulding operations including resin application on to the reinforcement fibre has to be completed before the point of gelation (Agrawal 1988; Rodriguez 1991; Shailesh and James 1991; Yang and Laurent 1991; Malkin et al 1997; Laza et al 1998; Cook et al 2001; Ishida and Douglas 2001; Vilas et al 2001; Abadie et al 2002; Rimduist and Ishida 2002; Han 2004; Lionetto et al 2004; Devillard et al 2005; Hossein et al 2005; Xing et al 2007). The nature of exotherm raised from the exothermic effect of curing resin determines volume shrinkage, warping, residual stress, degradation of polymer, smoke, resin cracking, etc. Hence, to achieve good quality product, the resin gelation and curing reaction has to occur in a controllable manner (Agrawal 1988; Rodriguez 1991; Cook et al 2001; Abadie et al 2002; Hossein et al 2005). The phase transformations and the heat evolving nature of curing resin can be altered with the mixing proportions of curing agents. Hence, a judicious choice of initiator and promoter proportions can avoid reduced gel-time and short time exothermic reactions (Rodriguez 1991; Shailesh and James 1991; Yang and Laurent 1991; Laza et al 1998; Cook et al 2001; Vilas et al 2001; Abadie et al 2002; Han 2004; 
(a)

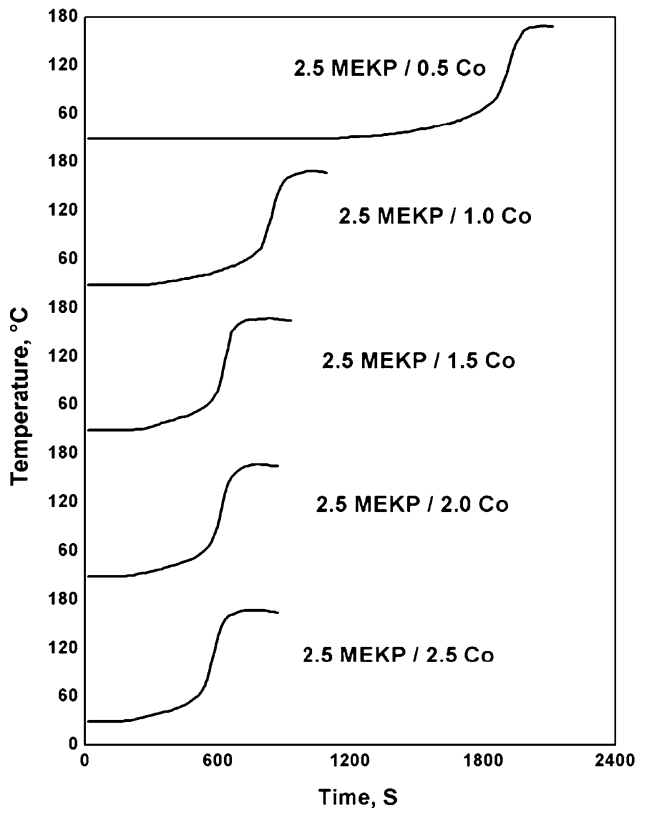

(b)

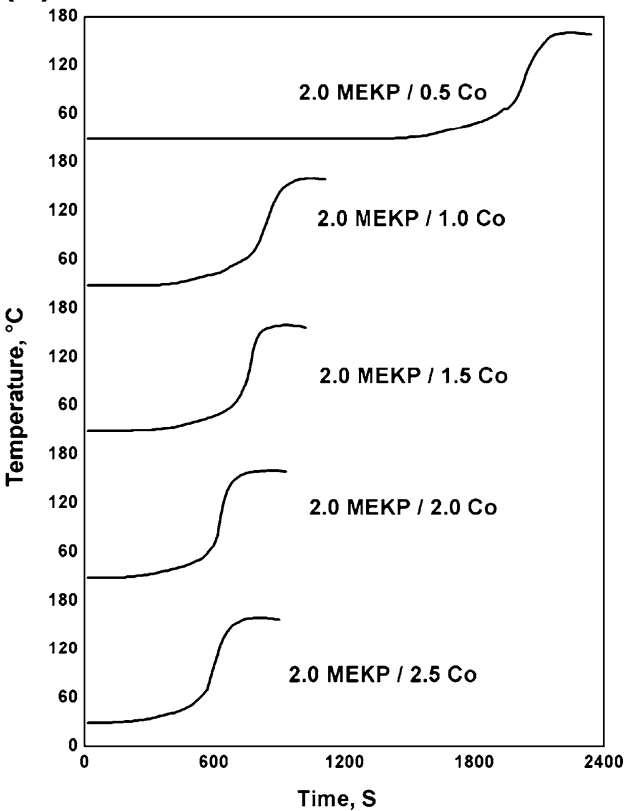

Figure 1. Measured exotherm of unsaturated polyester resin system at various catalyst and accelerator volume proportion.

Table 1. Effect of accelerator and MEKP volume ratio on gel time.

\begin{tabular}{lllllll}
\hline & & $2 \cdot 5$ & 2 & $1 \cdot 5$ & 1 & $0 \cdot 5$ \\
\cline { 3 - 7 } Accelerator (phr) & & \multicolumn{5}{c}{ Gel time (S) } \\
\hline MEKP (phr) & $2 \cdot 5$ & 227 & 238 & 249 & 384 & 1151 \\
& 2 & 244 & 290 & 360 & 416 & 1213 \\
& $1 \cdot 5$ & 248 & 305 & 372 & 448 & 2705 \\
& 1 & 754 & 975 & 1210 & 1655 & 6422 \\
& 0.5 & 1564 & 1776 & 2467 & 5028 & - \\
\hline
\end{tabular}

Devillard et al 2005; Hossein et al 2005; Penczek et al 2005; Xing et al 2007).

Experimental techniques such as the measurement of flow cessation time (as per ASTM D2471 test) (Agrawal 1988; Rodriguez 1991; Cook et al 2001; Abadie et al 2002; Hossein et al 2005; Xing et al 2007), the measurement of infinity shear viscosity time (viscometers and rheometers) (Yang and Laurent 1991; De La Caba et al 1997; Malkin et al 1997; Laza et al 1998; Vilas et al 2001; Abadie et al 2002; Rimduist and Ishida 2002; Lionetto et al 2004; Lionetto and Maffezzoli 2008) and the measurement appearance of equilibrium modulus time (dynamic mechanical analysers) (Shailesh and James 1991; Malkin et al 1997; Ishida and Douglas 2001; Vilas et al 2001; Rimduist and Ishida 2002; Lionetto et al 2004) have been utilized by many researchers to detect the time of resin gelation. The measurements of resin cure exotherm have been achieved by the use of thermocouples (as per ASTM D2471 test) (Agrawal 1988; Rodriguez 1991; Cook et al 2001; Abadie et al 2002; Hossein et al 2005). Researchers have qualitatively correlated the effect of initiator, promoter, inhibitor and applied cure temperature on gelation and exotherm behaviour (Rodriguez 1991; Shailesh and James 1991; Yang and Laurent 1991; Cook et al 2001; Vilas et al 2001; Abadie et al 2002; Han 2004; Devillard et al 2005; Hossein et al 2005; Xing et al 2007; Laza et al 1998). There have been a few more detailed analyses of the effect of the proportions of MEKP and cobalt octoate on the gel time and curing behaviour of unsaturated polyester resins (Rodriguez 1991; Cook et al 2001; Vilas et al 2001; Abadie et al 2002; Ling et al 2004; Lionetto et al 2004). In general, the modeling of gel time and exotherm parameters as a function of levels of initiator, promoter, inhibitor and temperature can be classified into two categories viz., mechanistic models and the phenomenological or empirical models. The mechanistic models attempt to quantify the balance of chemical species taking part in the cure reaction by using a mathematical function that relate the gel time and other exotherm parameters to concentrations of curing agents, inhibitors and applied cure temperatures (Agrawal 1988; Ramis and Salla 1999; Cook et al 2001; Han 2004). Phenomenological or empirical models are mathematical expressions which are 


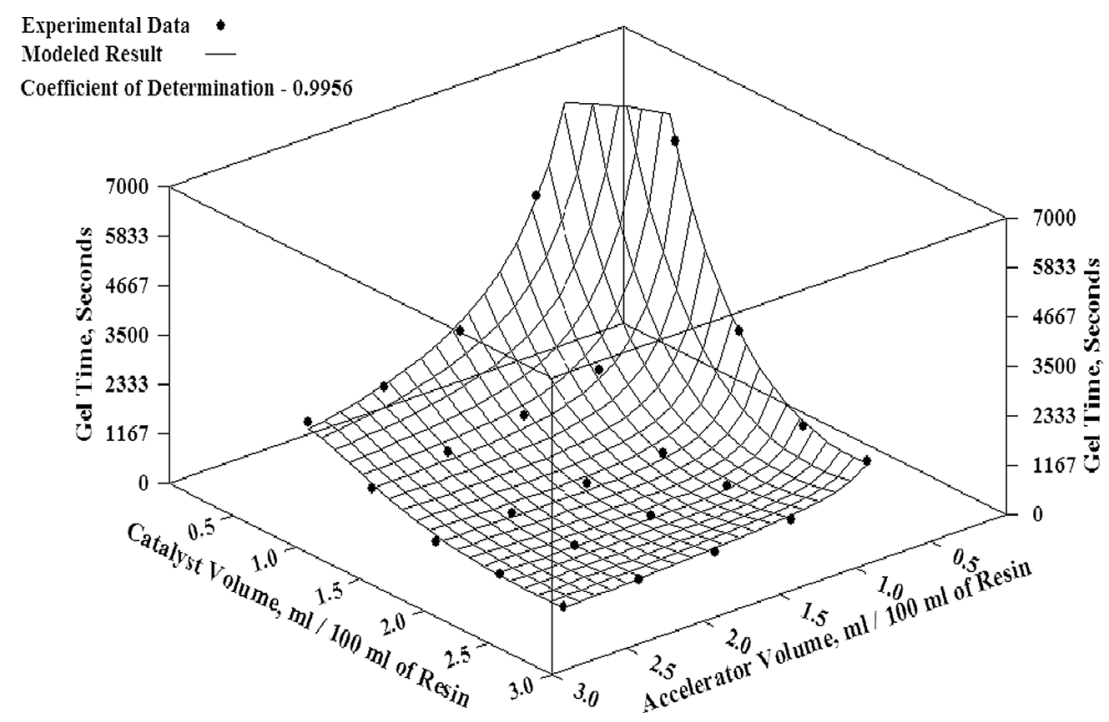

Figure 2. Gel time as a function of catalyst and accelerator volume proportions.

Table 2. Effect of accelerator and MEKP volume ratio on peak exotherm time.

\begin{tabular}{lllllll}
\hline & & $2 \cdot 5$ & 2 & 1.5 & 1 & 0.5 \\
\cline { 3 - 6 } Accelerator (phr) & & \multicolumn{5}{c}{ Peak exotherm time (S) } \\
\hline MEKP (phr) & $2 \cdot 5$ & 750 & 765 & 825 & 1055 & 2055 \\
& 2 & 780 & 840 & 915 & 1020 & 2220 \\
& 1.5 & 810 & 870 & 1005 & 2040 & 4155 \\
& 1 & 1410 & 1665 & 2055 & 2730 & 9765 \\
& $0 \cdot 5$ & 2550 & 2865 & 4170 & 8265 & - \\
\hline
\end{tabular}

framed to fit the dependence of gel time and other cure parameters in terms of curing aids proportions (Shailesh and James 1991; Devillard et al 2005) and these empirical models are easier to handle in comparison with mechanistic models.

The main objective of this work is to quantify and correlate the effects of varying initiator and promoter volume levels on gel time and exotherm behaviour of an unsaturated polyester resin using experimental and empirical curve fitting technique. This article is structured as follows: first, the exotherm obtained from the experiments on varying curing aids concentrations are presented. Then the shifts of gel time with change of initiator and accelerator proportions have been correlated using nonlinear regression analysis. Finally, the exotherm parameters such as peak exotherm temperature, peak exotherm time and rate of rise in temperature are profiled with the levels of cure ingredients dependence.

\section{Materials}

A commercially available medium reactive unsaturated polyester resin with a grade name ISO RTM was used in this study. It is tailored with a mixture of isophthalic acid, maleic anhydride and propylene glycol to an acid value of $15 \pm 2$ $\mathrm{mg} \mathrm{KOH} / \mathrm{g}$. It has the density of $1.080 \mathrm{~g} / \mathrm{cc}$ with $43 \%$ (w/w) volatile content. A thermal source or reduction-oxidation (redox) initiation system is often used for the curing of UP resin (Agrawal 1988; Rodriguez 1991; Shailesh and James 1991; Yang and Laurent 1991; Ramis and Salla 1999; Cook et al 2001; Vilas et al 2001; Lionetto et al 2004; Hossein et al 2005). For applications such as resin transfer moulding (RTM), UP resins are usually cured at ambient temperatures through the use of a redox initiation system composed of initiators/catalysts and promoters/accelerators (Shailesh and James 1991; Cook et al 2001; Vilas et al 2001; Hossein et al 2005). Methyl ethyl ketone peroxide (MEKP) is one of the most frequently used peroxide based initiator (Rodriguez 1991; Yang and Laurent 1991; Cook et al 2001; Vilas et al 2001; Abadie et al 2002; Lionetto et al 2004; Hossein et al 2005). Cobalt salt such as cobalt octoate is employed as the accelerator to decompose peroxide based initiators in inducing free radical co-polymerization during resin curing process (Rodriguez 1991; Yang and Laurent 1991; Cook et al 2001; Vilas et al 2001; Abadie et al 2002; Lionetto et al 2004). In this work, methyl ethyl ketone peroxide along with cobalt octoate was used as redox initiation system for curing the unsaturated polyester resin used. MEKP has $8 \%$ active oxygen content whereas cobalt octoate has $3 \%$ cobalt content. UP resin and curing agents were supplied by Mechemco Resins Pvt. Ltd., Mumbai, India. 


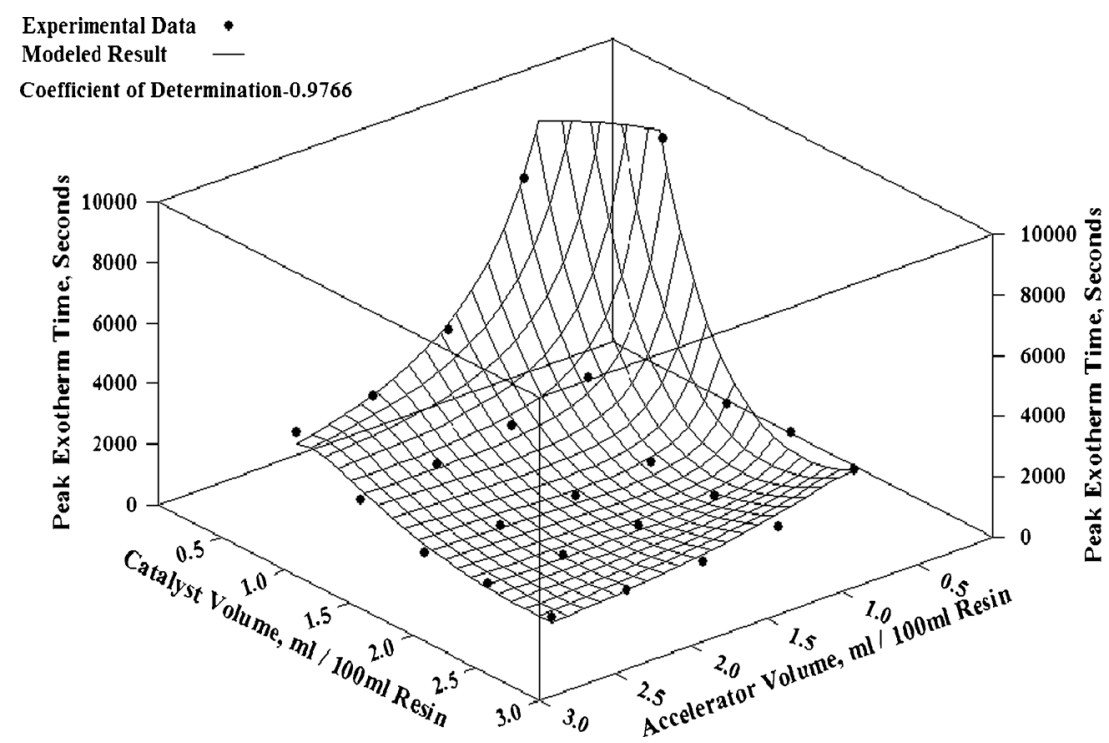

Figure 3. Peak exotherm time as a function of catalyst and accelerator volume proportions.

Table 3. Effect of accelerator and MEKP volume ratio on peak exotherm temperature.

\begin{tabular}{lllllll}
\hline & & 2.5 & 2 & 1.5 & 1 & 0.5 \\
\cline { 3 - 6 } Accelerator (phr) & & \multicolumn{5}{c}{ Peak exotherm temperature $\left({ }^{\circ} \mathrm{C}\right)$} \\
\hline MEKP (phr) & $2 \cdot 5$ & 167 & 167 & 168 & 169 & 169 \\
& 2 & 159 & 160 & 160 & 160 & 160 \\
& $1 \cdot 5$ & 157 & 156 & 156 & 156 & 155 \\
& 1 & 152 & 152 & 151 & 152 & 150 \\
& $0 \cdot 5$ & 147 & 147 & 146 & 145 & - \\
\hline
\end{tabular}

\section{Experimental}

The medium reactive unsaturated polyester resin was mixed with MEKP which acts as catalyst (initiator) and cobalt octoate which acts as accelerator (promoter) and allowed to cure. During curing, the important curing characteristics of this resin system such as gel time, peak exothermic temperature and peak exothermic time were measured at room temperature according to the specification ASTM D2471. Peak exotherm of the resin system was measured using various volume proportions of MEKP and cobalt octoate, which were performed to quantify effect of different concentration levels of initiator and promoter. Accelerator volume was varied from 0.5 to $2.5 \mathrm{~mL}$ and the initiator volume was varied from 0.5 to $2.5 \mathrm{~mL} / 100 \mathrm{~mL}$ of resin. Initially, $100 \mathrm{~mL}$ of the resin sample was added with desired proportion of accelerator and the mix was agitated slowly with a stirrer avoiding air entrapment. The timer was started when the initiator was added. A thermocouple was inserted into the geometric centre of the reacting mass and the temperature with time was recorded till the end of experiment. An applicator stick was inserted in the centre of the reacting mass for every $15 \mathrm{~s}$ and the flowability of the reacting mass was checked. The time elapsed from the start of mixing of initiator and when the reacting material no longer adhered to the end of a clean probe, it was recorded as gel time. The temperature with time was recorded continuously until the temperature started to drop. Highest temperature reached was recorded as the peak exothermic temperature and the elapsed time from the start of mixing to reach the maximum temperature was recorded as the peak exothermic time. The volume shrinkage due to resin cure and exotherm heat was measured using the change in volume between initial reacting resin volume and the final resin cast. The measurement of volume shrinkage helps to predict the shape distortions of a composite structure during resin cure. All the experiments were conducted at an ambient temperature of $25^{\circ} \mathrm{C}$.

\section{Empirical gel time and exotherm model}

Gel time and exotherm data obtained from the performed experiments were correlated to the volume proportions of initiator and promoter. A nonlinear regression analysis of all gel time and exotherm data was performed using datafit software to quantify the variation of these parameters with the volume proportions of accelerator and initiator. This correlation can be used as an important tool to predict the gel time, 


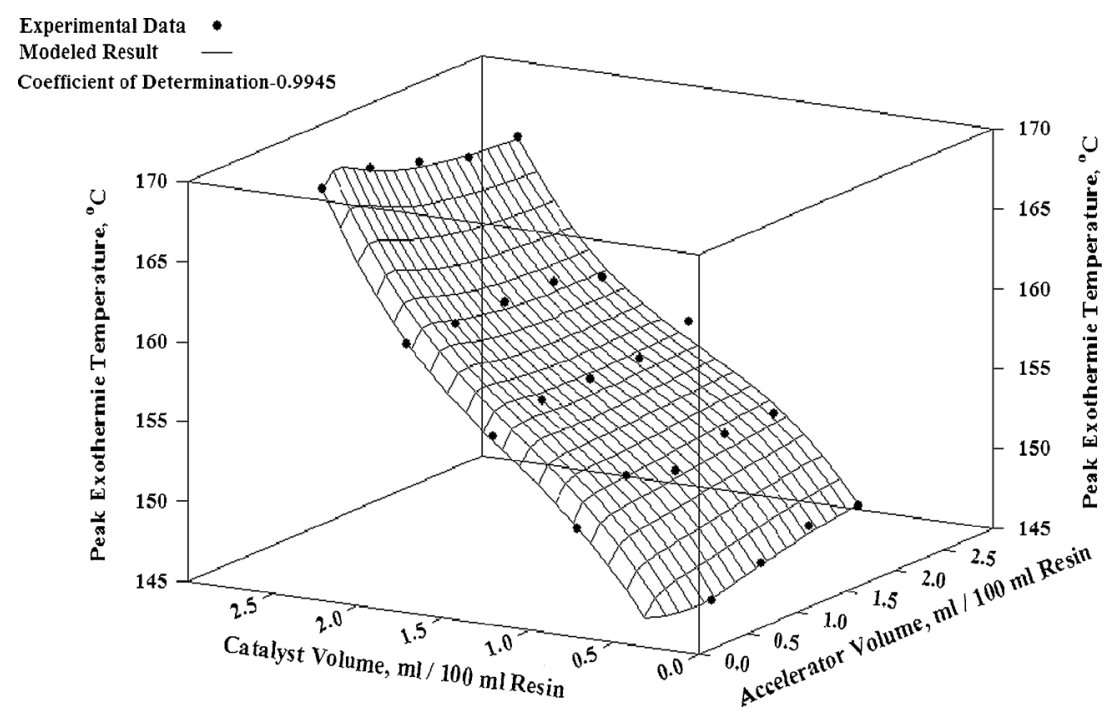

Figure 4. Peak exothermic temperatures as a function of catalyst and accelerator volume proportions.

Table 4. Effect of accelerator and MEKP volume ratio on rate of rise in temperature.

\begin{tabular}{lllllll}
\hline & & 2.5 & 2 & 1.5 & 1 & 0.5 \\
\cline { 3 - 7 } Accelerator (phr) & & \multicolumn{5}{c}{ Rate of temperature rise $\left({ }^{\circ} \mathrm{C} / \mathrm{min}\right)$} \\
\hline MEKP (phr) & 2.5 & 15.22 & 14.42 & 13.8 & 11.83 & 9.75 \\
& 2 & 12 & 11.56 & 11.3 & 10.83 & 9.45 \\
& 1.5 & 11.04 & 10.96 & 10.72 & 9.16 & 5.55 \\
& 1 & 10.38 & 9.76 & 8.34 & 6.1 & 2.7 \\
& 0.5 & 5.32 & 3.16 & 2.19 & 1.66 & - \\
\hline
\end{tabular}

peak exothermic temperature and peak exotherm time for medium reactive unsaturated polyester resin, MEKP initiator and cobalt octoate accelerator.

\section{Results and discussion}

\subsection{Effect of initiator and accelerator volume ratios on resin gel time and exotherm}

Figure 1 shows change in temperature with time during curing of the resin system at various initiator and accelerator volume proportions. These time-temperature curves show the nature of rise in temperature and the minimum time required for the resin cure with specific volume proportion of initiator and accelerator. From the measured exotherm data as shown in figure 1, it is evident that the use of higher proportions of initiator and accelerator yields reduced cure cycle time. Similar exotherm graphs can be produced for initiator proportion varying from 1.5 to $0.5 \mathrm{~mL}$ with accelerator proportion varying from 2.5 to $0.5 \mathrm{~mL} / 100 \mathrm{~mL}$ of the resin. The gel time and other curing parameters such as peak exotherm, peak exotherm time, rate of rise in temperature and volume shrinkage during curing reaction can be determined from the measured exotherm, which are tabulated in the subsequent sections. In all subsequent tabulations, the varying concentrations of accelerator and initiator to the resin parts are presented as "phr", which means the parts of accelerator and catalyst to hundred parts of resin.

\subsection{Effect of accelerator and MEKP volume ratio on gel time}

The empirical gel time data obtained from the measured exotherm are given in table 1. Figure 2 shows gel times measured for varying proportions of initiator and accelerator from 0.5 to $2.5 \mathrm{~mL} / 100 \mathrm{~mL}$ of resin. From the same figure, it is clearly observed that, for a particular initiator proportion, more the accelerator part, lesser the gel time. And for a particular accelerator proportion, more the initiator part, lesser the gel time. This fact is chiefly attributed to the increase in decomposition of peroxides due to increase in initiator concentration to produce highly reactive free radicals which in turn react with polymer molecules to produce cross links. Also, the gel time difference appears to be approximately low with high levels of accelerator and initiator. Using nonlinear regression analysis, gel times were fit as a function of initiator and accelerator volume proportions to the form as given by (1) and it is seen that the experimental 


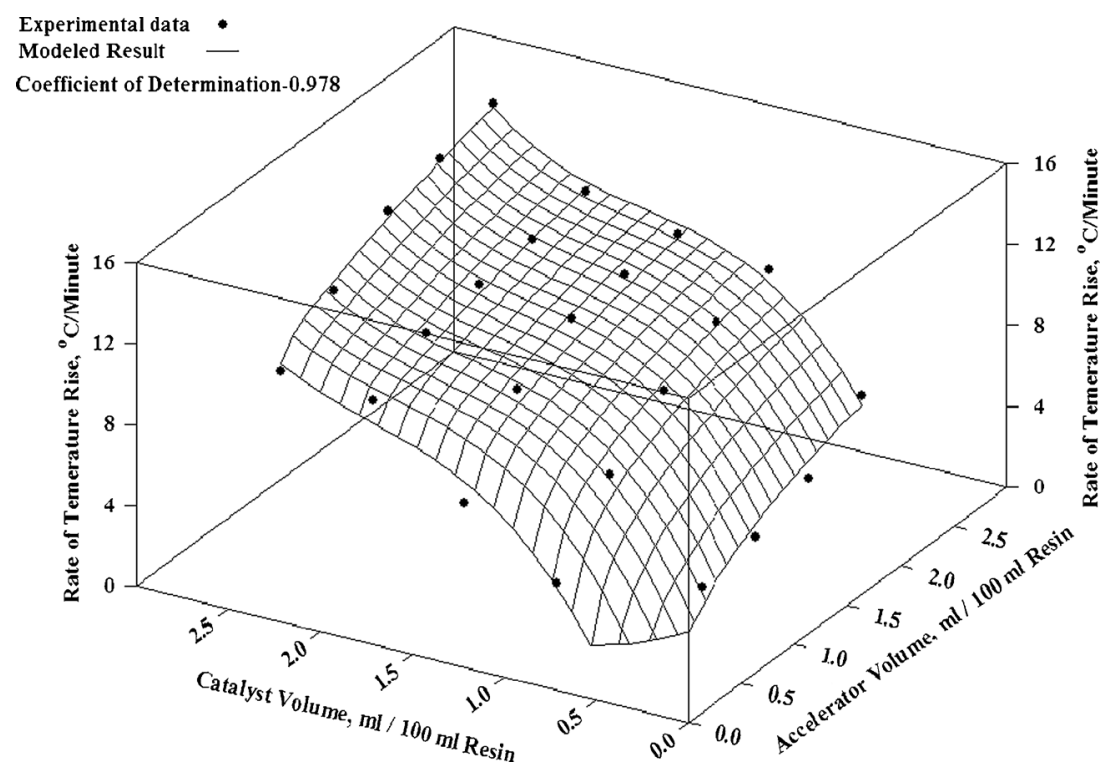

Figure 5. Rate of rise in temperature during reaction as a function of catalyst and accelerator volume proportions.

Table 5. Effect of accelerator and MEKP volume ratio on volume shrinkage.

\begin{tabular}{|c|c|c|c|c|c|c|}
\hline \multirow{2}{*}{\multicolumn{2}{|c|}{ Accelerator (phr) }} & $2 \cdot 5$ & 2 & 1.5 & 1 & $0 \cdot 5$ \\
\hline & & \multicolumn{5}{|c|}{ Volume shrinkage (\%) } \\
\hline \multirow[t]{5}{*}{ MEKP (phr) } & $2 \cdot 5$ & $10 \cdot 4$ & $10 \cdot 2$ & 10 & $7 \cdot 6$ & 6 \\
\hline & 2 & $8 \cdot 6$ & $8 \cdot 2$ & $8 \cdot 4$ & $7 \cdot 6$ & 7 \\
\hline & 1.5 & $8 \cdot 5$ & $8 \cdot 4$ & $8 \cdot 6$ & $7 \cdot 6$ & 7 \\
\hline & 1 & $7 \cdot 8$ & $7 \cdot 6$ & 8 & 7 & 6 \\
\hline & $0 \cdot 5$ & 7 & $6 \cdot 8$ & $6 \cdot 8$ & 6 & - \\
\hline
\end{tabular}

dependence fits accurately well to the empirically modelled equation, as shown in figure 2 :

$$
G=1694 * A^{-1.785} * C^{-1.682}
$$

where $G$ the gel time in seconds, $A$ the accelerator volume in $\mathrm{mL}$ and $C$ the initiator volume in $\mathrm{mL}$.

\subsection{Effect of accelerator and MEKP Volume ratio on peak exotherm time}

The empirical peak exotherm time data obtained from the measured exotherm are given in table 2. Figure 3 shows dependence of peak exotherm time measured on varying proportions of initiator and accelerator from 0.5 to $2.5 \mathrm{~mL}$ per $100 \mathrm{~mL}$ of resin. From the same figure, it is evident that, for a particular initiator proportion, more the accelerator part, lesser the peak exotherm time. And for a particular accelerator proportion, more the initiator part, lesser the peak exotherm time. Comparing figures 2 and 3 , it can be noticed that the gel time and the time to peak exotherm shows similar behaviour with respect to the variations of accelerator and initiator levels. A nonlinear regression analysis of peak exotherm time data correlating accelerator and initiator levels was performed and a comparison of actual and modelled results are given in figure 3 . The empirical form given in (2) gives a better definition in relating peak exotherm time to the volume proportions of the curing aids:

$$
P E_{t}=2832 * A^{-1.534} * C^{-1 \cdot 612} \text {, }
$$

where $P E_{t}$ is the peak exotherm time in seconds.

\subsection{Effect of accelerator and MEKP volume ratio on peak exotherm temperature}

The empirical peak exotherm temperature data obtained from the measured exotherm are given in table 3. The effect of varying the levels of initiator and promoter on the exotherm is displayed in figure 4. From the same figure, it can be inferred that for varying initiator level, the peak exotherm is unaffected on cobalt salt level from the accelerator. This may be caused by the non-consumption of metal salts during curing reaction, since the consumed metal salts for peroxides decomposition can be regenerated with the reaction progression. It can also be noted that, for a particular accelerator 


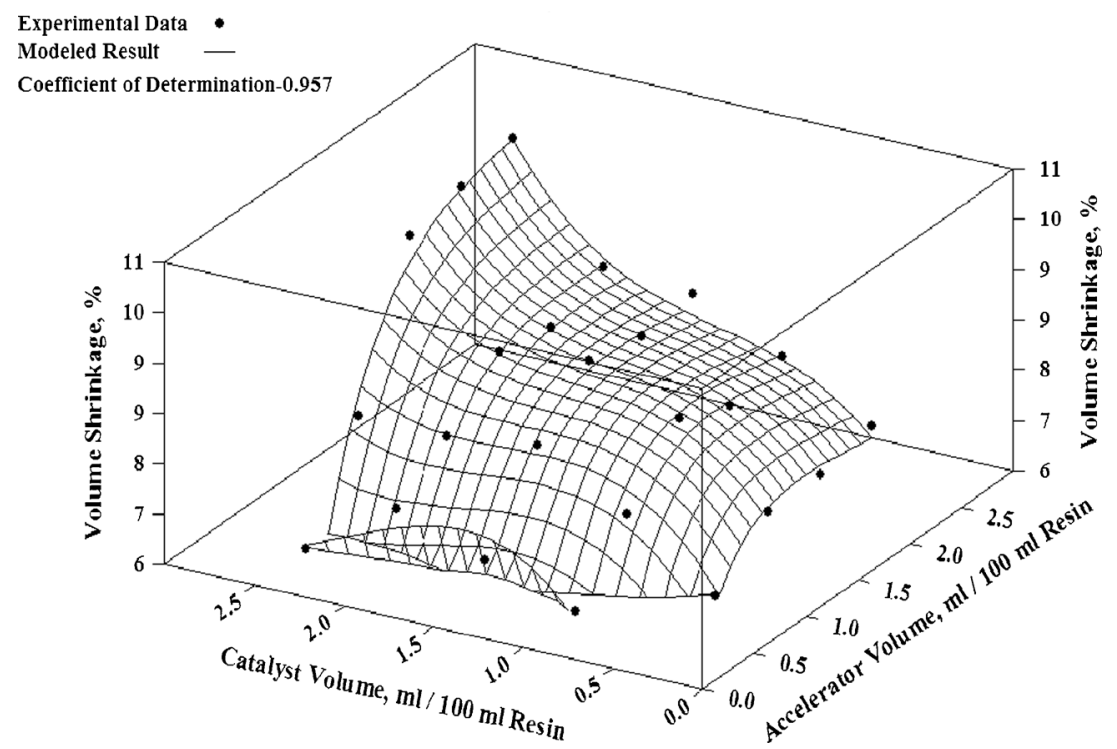

Figure 6. Volume shrinkage during curing reaction as a function of catalyst and accelerator volume proportions.

proportion, lesser the initiator part, lower the peak exothermic temperature. This trend is due to the depletion of initiator at the lower volume levels before full reaction. The peak exothermic temperature varies from 169 to $145^{\circ} \mathrm{C}$ with the initiator volume differing from 2.5 to $0.5 \mathrm{~mL} / 100 \mathrm{~mL}$ of resin. The empirical form given in (3) gives a better prediction of peak exothermic temperature using volume proportions of the curing aids:

$$
P E_{T}=152 \cdot 5 * A^{-0.003} * C^{-0.083},
$$

where $P E_{T}$ is the peak exothermic temperature in ${ }^{\circ} \mathrm{C}$.

\subsection{Effect of accelerator and MEKP volume ratio on rate of rise in temperature}

The empirical rate of rise in temperature data obtained from the measured exotherm are given in table 4 . The plot rate of rise in temperature after resin gelation with respect to varying initiator and accelerator volume levels is portrayed in figure 5. It can be observed from the same figure that there exists a quick rise in temperature with the use of higher proportions of initiator and accelerator volumes. The reason for this observation is a higher decomposition rate of initiator with higher proportion of accelerator. The effect of accelerator and initiator levels on the rate of rise in temperature during resin curing can be defined with an empirical form given by (4). Figure 5 shows comparison of the experimentally obtained temperature rise per unit time with that predicted by the empirical model:

$$
R T R=6 \cdot 17 * A^{0.31} * C^{0.72},
$$

where $R T R$ is the rate of rise in temperature ${ }^{\circ} \mathrm{C} / \mathrm{min}$.

\subsection{Effect of accelerator and MEKP volume ratio on volume shrinkage}

The empirical cure volume shrinkage data obtained from the measured exotherms are given in table 5. The impact of cobalt octoate and methyl ethyl ketone levels on cure volume shrinkage of a medium reactive unsaturated polyester resin is depicted in figure 6. As can be seen from the same figure, the volume shrinkage during curing reaction varies from 6 to $11 \%$ for the volume proportions of accelerator and initiator used. It may be mentioned that the volume shrinkage increases notably with the increase in the initiator in comparison with the accelerator part. The volume shrinkage can be correlated to the volumes of accelerator and initiator by fitting the experimental data to an empirical form using nonlinear regression analysis as given by (5)

$$
V S=6 \cdot 82 * A^{0.204} * C^{0.196},
$$

where VS is the volume shrinkage in \%.

\section{Conclusions}

The impact of cobalt octoate and methyl ethyl ketone volume ratios on gelation time and exotherm behaviour of an unsaturated polyester resin were studied. The resin gelation was found to correspond with the onset of temperature rise during resin curing and also the gelation time was found to decrease with increase in concentrations of accelerator and initiator. Results showed that the peak exotherm and rate of rise in temperature increase with increasing curing aids as a cause of higher decomposition rate of initiator. The use of nonlinear regression analysis helped to correlate the gel time data and exotherm data with volume proportions of cobalt octoate and methyl ethyl ketone, qualitatively and quantitatively. 


\section{References}

Abadie M J M, Mekhissi K and Burchill P J 2002 J. Appl. Polym. Sci. 841146

Agrawal J P 1988 Eur. Polym. J. 2493

Cook Wayne D, Lau Michelle, Mehrabi Mansour, Dean Katherine and Zipper Marcus 2001 Polym. Int. 50129

De La Caba, Guerrero P, Eceiza A and Mondragon I 1997 Eur. Polym. J. 3319

Devillard Antoine Laut and Suresh G Advani 2005 Polym. Compos. 2674

Han Tong-chun 2004 J. Univ. Sci. 5928

Hossein Beheshty M, Hassan Nasiri and Mehdy Vafayan 2005 Iranian Polym. J. 14990

Ishida H and Douglas J Allen 2001 J. Appl. Polym. Sci. 79406

Laza J M, Julian C A, Larrauri E, Rodriguez M and Leon L M 1998 Polymer 4035
Ling Li, Xia Cao and James Lee 2004 Polymer 456601

Lionetto F and Maffezzoli A 2008 Adv. Polym. Technol. 2763

Lionetto F, Rizzo R, Luprano V A M and Maffezzoli A 2004 Mater. Sci. Eng. A 370284

Malkin A Ya, Kulichikhin S G, Kerber M L, Gorbunova I Yu and Murashova E A 1997 Polym. Eng. Sci. 371322

Penczek P, Czub P and Pielichowski J 2005 Adv. Polym. Sci. 1841

Rimduist S and Ishida H 2002 Rheol. Acta 411

Rodriguez Ernesto L 1991 Polym. Eng. Sci. 311022

Salla R and Salla J M 1999 J. Polym. Sci. B: Polym. Phys. 37751

Shailesh V Muzumdar and James Lee 1991 Polym. Eng. Sci. 31 1647

Vilas J L, Laza J M, Garay M T, Rodriguez M and Leon L M 2001 J. Appl. Polym. Sci. 79447

Xing Cheng, Zhang S Y, James Deng and Siqun Wang 2007 J. Appl. Polym. Sci. 1031566

Yang Y S and Suspene L 1991 Polym. Eng. Sci. 31321 